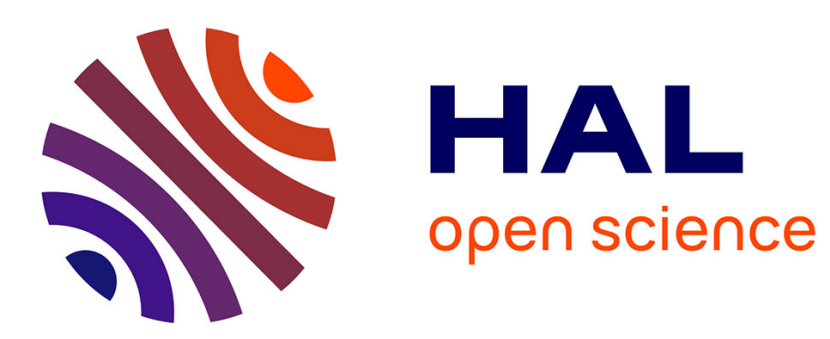

\title{
L'évaluation du prix des actions par les fondamentaux: analyse du marché français
}

Dominique Pepin

\section{To cite this version:}

Dominique Pepin. L'évaluation du prix des actions par les fondamentaux : analyse du marché français. Economie et Prévision, 2010, 195-196, pp.83-98. hal-00963639

\section{HAL Id: hal-00963639 \\ https://hal.science/hal-00963639}

Submitted on 21 Mar 2014

HAL is a multi-disciplinary open access archive for the deposit and dissemination of scientific research documents, whether they are published or not. The documents may come from teaching and research institutions in France or abroad, or from public or private research centers.
L'archive ouverte pluridisciplinaire HAL, est destinée au dépôt et à la diffusion de documents scientifiques de niveau recherche, publiés ou non, émanant des établissements d'enseignement et de recherche français ou étrangers, des laboratoires publics ou privés. 


\title{
L'évaluation du prix des actions par les fondamentaux : \\ analyse du marché français
}

\author{
Dominique Pépin ${ }^{(*)}$
}

Depuis 1920, le prix des actions en France, comme partout dans le monde, a connu d'importantes variations. Leurs conséquences dommageables sur l'activité économique réelle ont conduit à s'interroger sur le rôle des banques centrales, et à se demander si ces dernières ne devaient pas prendre en compte le prix des actifs financiers dans leurs objectifs. Mais à quelle valeur de référence peuvent-elles comparer le prix effectif des actions? Nous proposons comme benchmark une modélisation du prix du capital, dépendant du partage de la valeur ajoutée et de la productivité apparente du capital (les variables fondamentales), et faisant des profits agrégés la composante essentielle du prix des actions. Le prix réel des actions dépend alors de l'actualisation du taux de rendement réel du capital (le profit réel d'une unité de capital), c'est-à-dire le produit du taux de marge et de la productivité.

La stabilité en longue période des variables fondamentales et leur comportement " markovien " permettent d'écrire que le prix réel du capital est une fonction linéaire du profit réel courant. Dans la longue période, le prix réel du capital définit une constante, le prix naturel du capital, dont il ne peut s'éloigner en courte période que si les fondamentaux s'écartent eux-mêmes de leurs valeurs de longue période.

Nous confrontons la valeur observée d'un indice du marché français à sa valeur fondamentale décrite par le modèle. Des écarts importants sont observés, attestant que les variables fondamentales, si elles ont un effet significatif sur le prix du capital, n'expliquent pas la majeure partie de ses variations. Nous observons que le marché français est soumis à des cycles longs, dont la durée se mesure en décennies, et dont les bulles spéculatives ne constituent que la partie la plus visible. Le marché français aurait connu trois de ces cycles depuis 1920, la naissance du cycle actuel remontant au début des années 80. Durant ces cycles, le prix des actions connaît une phase d'appréciation, associée à une valorisation excessive du prix des actifs au regard des profits, puis une phase de baisse associée à la correction et à la sous-évaluation.

L'importante volatilité des prix par rapport aux fondamentaux produit une estimation très variable du prix naturel du capital. L'incertitude entourant l'estimation de cette constante de longue période (son intervalle de confiance à un niveau de $95 \%$ varie du simple au double), en dépit de l'utilisation d'une chronique longue d'un siècle, démontre qu'il n'est guère possible d'estimer de façon fiable la valeur fondamentale des actions. La durée des cycles interdit par ailleurs d'attribuer les écarts de prix à des erreurs d'appréciation temporaires des agents. A moins de croire que les agents puissent se tromper durablement, des mécanismes déstabilisateurs puissants, liés au crédit et à l'endettement, sont certainement à l'origine de ces cycles. Toute politique monétaire à destination des marchés financiers, qui ne corrigerait pas les déséquilibres liés au crédit et à l'endettement, ne produirait sans doute que des effets temporaires sur le prix des actifs, le symptôme persistant si la cause demeure.

\footnotetext{
${ }^{(*)}$ CRIEF, Université de Poitiers.

E-mail : dominique.pepin@univ-poitiers.fr

L'auteur remercie les rapporteurs anonymes pour leurs nombreuses remarques et suggestions pertinentes.
} 
L'influence de l'instabilité financière sur l'activité économique réelle est un sujet de recherche récurrent en économie, qui fait écho aux événements financiers de chaque époque et se trouve régulièrement remis au premier plan de notre attention. En période de turbulences financières, les mêmes questions sont posées; et chacun de s'inquiéter des conséquences de la crise, de s'interroger quant à la façon d'en juguler les effets, et de se demander comment faire pour que de tels événements ne surviennent plus jamais.

De ces diverses interrogations, la dernière est sans doute la plus simple, tant il saute aux yeux de l'historien de la finance que les crises sont inhérentes au fonctionnement de nos économies, et qu'il est impossible d'y remédier (Galbraith, 1989 et 1992 ; Kindleberger, 1978). C'est toujours la même histoire qui se répète. Dans la phase d'expansion, le crédit progresse beaucoup plus rapidement que la production, l'aisance en liquidité contribuant à alimenter une euphorie spéculative. Les agents s'enrichissent grâce à la valorisation excessive de leur patrimoine financier, l'effet d'accélération financière venant soutenir et démultiplier la phase expansive initiale. Cette dynamique déséquilibrante, par les distorsions qu'elle provoque dans les prix des actifs, fragilise les bilans des agents, et trouve toujours une issue fracassante dans une correction brutale des prix. S'ensuit une phase dépressive, symétriquement inverse à la phase d'expansion, qui ne trouve sa fin que lorsque les agents ont reconsolidé leurs bilans.

Les cycles financiers, dont l'existence ne peut être niée, trouvent chez les économistes différentes origines selon leur degré d'attachement à la théorie de l'efficience des marchés de capitaux. Sont-ils des cycles d'équilibre résultant de variations dans les "fondamentaux », ou sont-ils des cycles de déséquilibre résultant d'une interaction entre le crédit et l'évaluation financière, notifiant un écart parfois durable entre le prix des actifs financiers et celui qu'ils devraient afficher? Si la seconde réponse est la bonne, alors il apparaît avec force que la « finance n'est pas neutre » (Aglietta, 2008), et l'existence de ces cycles financiers devrait être une préoccupation de premier plan.

Les chercheurs en économie monétaire se sont particulièrement préoccupés de cette question, se demandant si la politique monétaire devait prendre en compte le prix des actifs. La sensibilité des entreprises et des établissements de crédit aux prix des actifs (canal large du crédit et canal du capital bancaire) justifie que ces prix puissent être pris en compte dans certaines circonstances (Pollin, 2005). Les banquiers centraux ont pourtant repoussé à ce jour le principe de l'introduction de la stabilisation des prix d'actifs dans la définition de leurs objectifs finaux. Deux motifs essentiels expliquent ce rejet (Artus, 2001). D'abord, la perspective de soutenir les marchés en cas de baisse excessive des prix leur déplaît fortement. Ce serait s'assujettir aux marchés, et ouvrir la porte à tous les excès. Ensuite, même s'il leur convenait de mettre en œuvre une telle politique, comment pourrait-elle être appliquée si on ne sait pas comment évaluer correctement les actifs ? Car toute politique de ce type requiert une théorie normative des prix, afin de substituer une évaluation individuelle (celle du banquier central) à celle des marchés.

Pourtant, une évaluation objective des actifs est nécessaire à l'établissement de normes comptables, si l'on veut encadrer l'activité des banques et autres intermédiaires financiers. Le principe de la fair value, qui valorise à la valeur de marché les actifs détenus par ces établissements, rend particulièrement instable l'évaluation de leurs bilans. Une politique prudentielle adéquate requiert l'établissement de normes d'évaluation des actifs, qui puissent dans l'idéal servir de guide aux investisseurs, ou de façon plus pragmatique qui puissent fournir des principes de valorisation comptable des actifs, tout en limitant les effets procycliques liés à l'instauration des contraintes réglementaires de fonds propres.

Si l'on accepte l'idée que les marchés financiers peuvent faillir à leur tâche d'évaluation des actifs financiers, reste à établir une théorie des prix qui puisse servir de guide. La théorie financière possède sur ce thème une accumulation de nombreux travaux, dont la plus grande partie est cependant consacrée, non pas à l'évaluation du prix des actifs, mais à celle de la rentabilité espérée ou de la prime de risque. En théorie, passer de l'évaluation de la prime de risque à celle des prix est simple. En pratique l'équivalence paraît beaucoup moins évidente. D'ailleurs, les travaux statistiques et économétriques portent quasi-exclusivement sur des données de rentabilité, et plus rarement de prix. Inéluctablement, tout travail sur des données de prix d'actions renvoie à la formule d'actualisation des dividendes de Williams (1938), dans laquelle les modèles d'évaluation financière modernes ne 
servent qu'à définir le taux utilisé pour actualiser le flux espéré des dividendes futurs aléatoires.

Le modèle d'actualisation des dividendes n'est cependant pas le seul modèle d'évaluation disponible. Le modèle d'actualisation des bénéfices constitue un concurrent sérieux, si l'on en juge par son emploi dans la littérature financière. A ces deux modèles, qui sont aujourd'hui les plus connus, nous avons préféré l'utilisation d'un troisième, qui se trouve en fait être plus ancien que le modèle de Williams, ses origines se perdant dans l'histoire de la théorie du capital: il s'agit du modèle d'actualisation des profits $^{(1)}$. L'intérêt de ce modèle, par rapport aux deux autres, est qu'il se prête beaucoup mieux à une analyse normative, et qu'il dépend beaucoup moins des anticipations d'un futur éloigné : l'influence du profit courant sur le prix du capital est bien plus importante que celle du dividende ou du bénéfice courant.

Ce papier se propose de montrer comment on peut utiliser ce modèle pour évaluer fondamentalement le prix des actions, grâce à des données macroéconomiques issues de la comptabilité nationale, et applique le principe obtenu sur le marché des actions françaises.

Dans une première partie, nous expliquons l'heuristique du modèle d'actualisation des profits, montrons en quoi la productivité apparente du capital et le taux de marge (la part de la valeur ajoutée consacrée à la rémunération du facteur capital) sont les déterminants fondamentaux du prix du capital d'une économie. L'analyse historique de la productivité et du taux de marge livre des enseignements essentiels sur le prix du capital dans la longue période ${ }^{(2)}$. Dans une deuxième partie, le modèle est présenté sous sa forme mathématique, et livre une formule d'actualisation dont on peut dire que c'est une formule d'évaluation fondamentale du prix des actions. Dans une troisième partie, un travail statistique permet de dégager un premier constat sur le prix réel des actions françaises dans la longue période. Théoriquement, les profits agrégés constituent une force de rappel du prix des actions. Puisqu'ils sont constants dans la longue période, il doit en être de même du prix réel des actions. La moyenne de ce dernier doit converger vers une constante de longue période qui peut alors servir de "phare» à l'évaluation des actifs. Or si l'existence de cette constante ne peut être remise en cause, il ressort que sa valeur est très mal identifiée, et que le « phare " guide les investisseurs de façon assez inefficace. Les profits agrégés semblent constituer une force de rappel assez lâche. L'analyse du prix réel des actions permet aussi de rendre compte de l'existence de cycles financiers longs. Depuis le début des années vingt, le marché français aurait connu trois de ces cycles. Ces derniers ont-ils une origine purement fondamentale ou sont-ils la manifestation de déséquilibres durables? La réponse se trouve dans la quatrième partie, où les variations du prix réel du capital sont étudiées dans la courte période. Il apparaît alors que même si une partie des variations de prix doit être attribuée aux variations des fondamentaux, l'explication de la majeure partie de ces variations est non fondamentale.

\section{Les facteurs fondamentaux du prix du capital: la productivité et le partage de la valeur ajoutée}

L'analyse que nous présentons peut être faite à tout niveau, celui de la firme ou du secteur qui intéresse plus l'analyste financier, ou celui du marché qui intéresse plus le macroéconomiste financier. C'est une lecture au niveau macroéconomique $^{(3)}$ que nous adoptons dans ce papier. Par souci de simplification, nous faisons l'hypothèse qu'un bien capital homogène est employé dans la production des biens et services, en association avec l'autre facteur de production, le travail. En ce sens, nous pouvons parler du prix (unique) du capital. Le capital étant homogène, et une action constituant un droit sur un stock de capital physique, le prix du portefeuille de marché des actions, ou d'un indice représentatif, correspond au prix du capital sur le marché considéré.

En termes réels, le capital vaut un certain nombre d'unités de biens et services produits. Le souci d'homogénéité et de cohérence avec la comptabilité nationale conduit à retenir le principe d'une valeur ajoutée homogène. Les facteurs de production de toute l'économie, travail et capital, produisent de la valeur ajoutée (dont la somme définit le PIB). Et cette valeur ajoutée vient rétribuer les facteurs de production, au terme d'un partage entre détenteurs du capital et salariés. Une unité de capital installée en début d'année permet à son propriétaire de réaliser dans l'année un profit, c'est-à-dire d'acquérir un certain nombre d'unités de valeur ajoutée. L'année suivante, cette unité de capital, dépréciée compte tenu de l'obsolescence, contribuera encore à la 
production, dégageant un peu moins de valeur ajoutée, dont une partie viendra la rémunérer. L'année suivante, elle permettra de gagner encore une part moindre de valeur ajoutée, etc. De la sorte, la détention d'une unité de capital donne droit à la perception d'un flux décroissant ${ }^{(4)}$ de profit (réel), les éléments anticipés de ce flux étant actualisés. La somme actualisée de cette série d'annuités anticipées définit le prix réel du capital, ou prix du capital par rapport au prix de la valeur ajoutée ${ }^{(5)}$.

Le prix réel du capital devrait ainsi résulter de l'actualisation du flux de valeur ajoutée captée par les détenteurs du capital, c'est-à-dire le rendement réel du capital (le profit réel par unité de capital). Il devient alors possible de procéder à une évaluation fondamentale du prix du capital en modélisant ce flux de profit. Une telle évaluation peut être faite à deux niveaux. Le prix du capital dépendant du partage de la valeur ajoutée, il est d'une part possible d'évaluer le prix du capital sur la base du partage observé de la valeur ajoutée, mais d'autre part il est aussi possible de donner une évaluation du prix du capital sur la base d'un partage équitable de la valeur ajoutée. Ce second niveau d'évaluation, aux prétentions normatives plus élevées ${ }^{(6)}$, présente des difficultés que nous n'avons pas essayé de résoudre, la tâche paraissant insurmontable. L'équilibre entre salaires et profits est complexe à déterminer, et de l'affrontement entre les tenants des diverses théories de ce partage ne s'est encore dégagé aucun consensus. Aussi nous en sommes-nous tenus à l'attitude qui consiste à rester en dehors de ce débat, le prix du capital étant formalisé sur la base du partage observé du revenu entre salariés et détenteurs du capital, sans jugement aucun des inégalités que ce partage pourrait produire.

Fondamentalement, le prix du capital par rapport au prix de la valeur ajoutée résulte de l'actualisation de la part du flux de valeur ajoutée produite par une unité de capital, captée par les détenteurs de capitaux. La formule d'évaluation qui en résulte dépend en conséquence des deux composantes suivantes : la productivité apparente du capital et le taux de marge, dont il faut modéliser les valeurs futures anticipées par des agents rationnels. Tout modèle d'évaluation dépend fortement de la modélisation des anticipations des variables futures impliquées, et reste dépendant de la pertinence de l'hypothèse de rationalité de ces anticipations. Plus ces variables futures connaissent des évolutions complexes et incertaines, plus il semble que la crédibilité de l'hypothèse d'anticipations rationnelles soit difficile à défendre. Si au contraire ces variables sont relativement inertes et connaissent des variations limitées, alors il devient plus facile de les prévoir, et les anticipations qui en résultent sont beaucoup plus proches de ce que seraient des anticipations rationnelles. Par chance, c'est le cas de la productivité apparente du capital et du taux de marge. La relative stabilité dans le temps de ces quantités fait d'ailleurs partie des faits stylisés énumérés par Kaldor (1963), qu'il pensait caractéristiques de la croissance économique. Kaldor (1963) a notamment énuméré les faits suivants: le rapport du capital physique à la production est approximativement constant, ainsi que les parts respectives du travail et du capital dans le revenu national. Si cela est vrai, alors le flux actualisé de profits anticipés peut être estimé avec une grande précision, et rend pertinent d'un point de vue pratique la formule de prix qui en résulte.

A cet effet considérons l'évolution tendancielle de la productivité apparente du capital. Rappelons que le volume de capital physique disponible à un moment donné dans une économie n'est pas observable directement. Le calcul de ce volume doit être reconstitué à partir de séries longues d'investissement du secteur privé (hors logement), selon une formulation qui dépend d'hypothèses relatives à la durée de vie des équipements. Le passage du volume de l'investissement au stock de capital nécessite de formuler des hypothèses sur la loi de mortalité des équipements, qui conditionnent le calcul du volume et en conséquence celui de la productivité apparente du capital.

A partir des données de production et du capital du Cepii, nous avons reconstitué( ${ }^{(7)}$ l'évolution de cette productivité en France sur une période d'un siècle : 1890-1985 (figure 1). Bien sûr, l'évolution de la productivité du capital doit être commentée avec prudence, mais en dépit des limitations statistiques, il ressort que la productivité du capital n'obéit à aucune tendance évidente (croissante ou décroissante). La série semble stationnaire. Tout au long du siècle, le rapport $Y / K$ a cependant connu d'importantes évolutions, qui permettent de tordre le cou à l'idée reçue que la productivité serait constante en courte période. En longue période, la constance de cette productivité semble toutefois une hypothèse raisonnable, comme le montre l'absence de tendance sur la figure 1. En 1890, la productivité du capital valait 0,48. En 1985, elle valait 0,43. Ce constat d'absence de tendance dans l'évolution de la productivité du capital est confirmé par d'autres 
auteurs (Dubois, 1985), et n'est d'ailleurs pas limité au cas de la France (Maddison, 1995).

Figure 1 : productivité du capital en France de 1890 à 1985

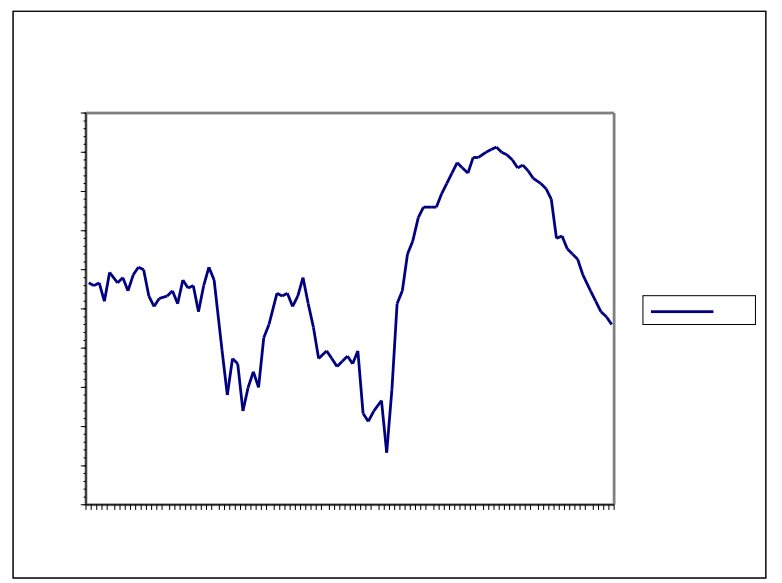

Pour les besoins de l'analyse économétrique présentée dans la section 3, nous avons construit nos propres données annuelles de capital sur la période 1970-2002. Nous avons estimé le volume de capital sur la période 1970-2002, à partir de la série de FBCF en volume ${ }^{(8)}$, en postulant une loi de mortalité soudaine au bout de 12 ans, avec amortissement linéaire ${ }^{(9)}$. Nous avons ensuite rapporté la série ainsi estimée de capital à la série de valeur ajoutée (en volume) pour former le rapport de productivité apparente de capital (figure 2).

Figure 2 : productivité du capital en France de 1970 à 2002

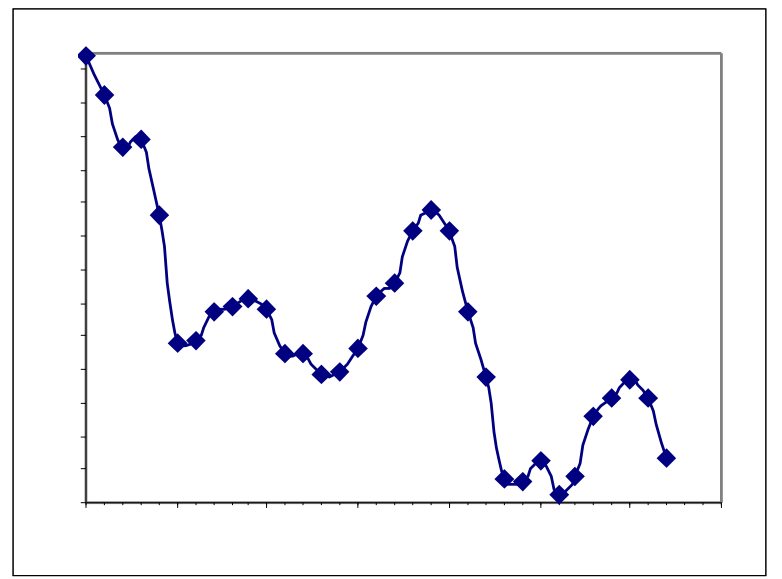

Le taux de marge, ou part du revenu du capital dans le produit intérieur brut, dont la valeur résulte du conflit d'intérêts qui oppose salariés et détenteurs des capitaux dans le partage de la valeur ajoutée, constitue l'autre variable déterminante $\mathrm{du}$ prix $\mathrm{du}$ capital. L'analyse historique du taux de marge a été entreprise par Prigent (1999), Sylvain (2001), Cette et Sylvain (2001) et Timbeau (2002). Il faut préciser que selon le traitement des données de base, issues de la comptabilité nationale, diverses méthodologies de calcul des taux de marge sont possibles ${ }^{(10)}$. L'extraction des taux de marge des données de comptabilité nationale constitue plus un travail d'estimation qu'un simple travail comptable, dont il faut garder à l'esprit qu'il n'est pas exempt de biais (Cette et Sylvain, 2001). Les diverses analyses soutiennent le principe que ce taux serait constant dans la longue période, en dépit de variations sensibles dans la courte période. Nous pouvons constater, à partir de données françaises (sources: Sylvain, 2007), que le partage de la valeur ajoutée entre les revenus du capital et du travail ne semble obéir à aucune tendance non stationnaire (figure 3). Certes, en courte période, le taux de marge semble soumis à des variations conjoncturelles non négligeables, mais leur cumul ne semble pas produire de tendance haussière ou baissière.

Figure 3 : taux de marge du secteur marchand (non agricole) en France

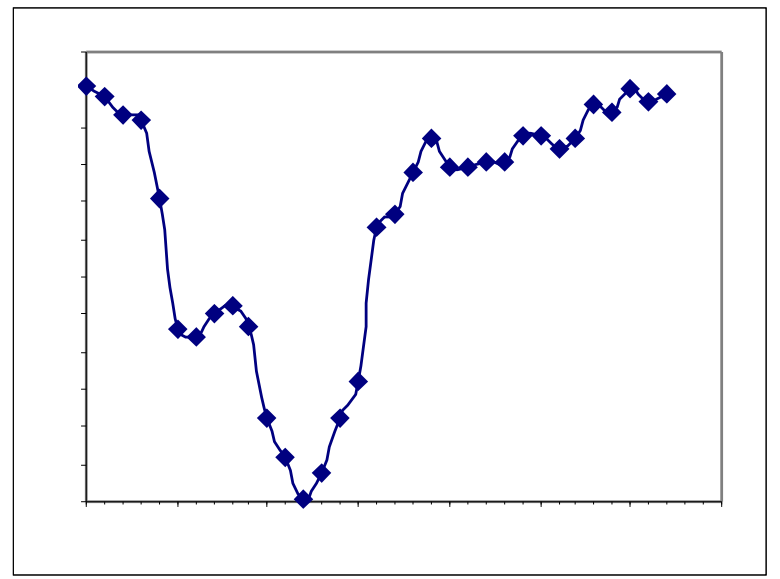

Le partage de la valeur ajoutée en France a connu une évolution très « contrastée » (Timbeau, 2002). Cette évolution, marquée par une forte inertie statistique, conduit d'ailleurs Prigent (1999) à accepter l'hypothèse de non stationnarité de la part des salaires, mais c'est une non-stationnarité en variance ${ }^{(11)}$, qui n'enlève rien de sa crédibilité à l'hypothèse de constance de l'espérance. Comme le fait remarquer Askenazy (2007), le taux de marge en France comme aux Etats-Unis était globalement en 2000 égal à sa valeur du siècle dernier. La constance sur longue période de ce taux peut être considérée comme l'une des constantes de la macroéconomie.

Ce constat n'est pas limité au cas de la France. Sur données américaines, Denison (1974, annexe J) et Jorgenson, Gollop, et Fraumeni (1987, tableau 9.3.) ont fourni des indications de la stabilité en longue période des parts du revenu américain allant aux facteurs de production. Ce constat est aussi confirmé par Sylvain (2001) et 
Cette et Sylvain (2001). De 1965 à 1999, le taux de marge a connu certes quelques variations aux Etats-Unis, qui peuvent faire dire que la constance en courte période de ce taux ne serait qu'une fable, mais néanmoins le cumul de ces variations est quasi-nul.

La productivité et le taux de marge étant constants dans la longue période, le profit réel l'est aussi (puisqu'il est égal à leur produit), et il doit en être de même en conséquence du prix réel du capital. Cette première implication est très importante, car elle stipule l'existence d'un prix naturel du capital au sens marshallien du terme, et qui fera l'objet d'une vérification dans la troisième section. Ce prix naturel désigne une constante de longue période, à laquelle le prix actuel du capital peut être comparé. Des écarts au prix naturel ne sont autorisés en courte période que si le profit s'écarte de sa propre valeur de longue période.

\section{Le modèle d'actualisation des profits}

Partons de l'identité comptable, où $Y_{t}$ représente la production en volume (somme de la valeur ajoutée) de l'économie considérée, $P_{t}$ le prix de la valeur ajoutée, $K_{t}$ le stock global de capital en volume, $r_{t}$ le rendement (nominal) du capital (c'est-à-dire le profit que dégage une unité de capital), et $W_{t}$ le salaire nominal versé à l'ensemble des travailleurs :

(1) $P_{t} Y_{t}=W_{t}+r_{t} K_{t}$

La valeur ajoutée produite par le travail et le capital $\left(P_{t} Y_{t}\right)$ fait l'objet d'un partage entre salaires $\left(W_{t}\right)$ et profits $\left(r_{t} K_{t}\right)$. Nous désignons par $q_{t}=r_{t} K_{t} /\left(P_{t} Y_{t}\right)$ la part des profits (la rémunération du capital) dans la valeur ajoutée. Sur 1 euro de revenu national, $q_{t}$ euro revient au capital. En termes réels, $q_{t}$ désigne aussi le pourcentage de la production (en volume) que l'ensemble des propriétaires des biens capitaux vont acquérir en $t$ grâce à la réalisation du profit.

L'ensemble du capital, d'un volume égal à $K_{t}$, donne à ses propriétaires le droit de percevoir la quantité $q_{t} Y_{t}$, prélevée sur l'ensemble de la production. Une unité de capital installée en début de période donne en conséquence droit à la perception sur toute la période de $q_{t} Y_{t} / K_{t}$ unité(s) du bien produit. Le profit réel d'une unité de capital est alors défini comme le produit du taux de marge $q_{t}$ et de la productivité apparente $\mathrm{du}$ capital $A_{t}=Y_{t} / K_{t}$. D'après les définitions posées, ce profit réel correspond à la valeur déflatée du profit nominal : $q_{t} A_{t} \equiv r_{t} / P_{t}$.

Notons $1-\delta_{k}$ le taux de dépréciation du capital au bout de $\mathrm{k}$ périodes après l'installation du capital, de sorte qu'au bout de $\mathrm{k}$ périodes, il reste d'une unité de capital la quantité $\delta_{k}<1$. D'une unité de capital installée en $t$, il reste ainsi une quantité résiduelle $\delta_{1}$ au bout d'une période, $\delta_{2}$ au bout de 2 périodes, etc. La suite des $\delta_{k}$ est décroissante et tend vers 0 .

Si on suppose que le capital anciennement installé et celui nouvellement installé possèdent la même productivité (après prise en compte de l'obsolescence et de l'usure), alors en $\mathrm{t}+1$ une unité résiduelle de capital ancien engendre le même profit qu'une unité nouvellement installée, c'est-à-dire $q_{t+1} A_{t+1}$. Mais d'une unité installée en $\mathrm{t}$, il ne reste en $\mathrm{t}+1$ que la quantité résiduelle $\delta_{1}$, qui offre le profit réel $\delta_{1} q_{t+1} A_{t+1}$. De la même façon, une unité de capital installée au début de la période $\mathrm{t}$ permet de percevoir sur la période $\mathrm{t}+2$ un profit égal à $\delta_{2} q_{t+2} A_{t+2}$, etc. Une unité de capital installé à la date $\mathrm{t}$ permet ainsi de percevoir le flux de profits réels $\left\{\delta_{k} q_{t+k} A_{t+k}\right\}_{k=0}^{\infty}$, $\operatorname{avec} \delta_{0}=1$.

Le prix réel d'une unité de capital installé en $\mathrm{t}$ correspond alors à la valeur réelle actualisée de ce flux. Plus précisément, ce flux étant incertain, compte tenu du caractère stochastique des variables $q_{t+k}$ et $A_{t+k}$ pour $k>0$, le prix réel d'une unité de capital doit être égal à l'espérance de la somme de ces éléments actualisés, l'actualisation se faisant à un taux qui tient compte du risque.

Notons $P_{k t}$ le prix d'une unité de capital installé au début de la période t. Le prix réel de cette unité est défini par $P_{k t} / P_{t}$. Ce rapport désigne le montant de biens produits que l'on peut acquérir en $t$ grâce au produit de la vente d'une unité de capital. A l'équilibre, le propriétaire d'une unité du bien capital doit être indifférent entre les deux options suivantes : vendre son unité de capital et acheter avec le produit de cette vente $P_{k t} / P_{t}$ 
unités de biens, ou conserver son unité de capital et consommer ultérieurement le flux de biens auquel donne droit cette unité.

Notons $i_{\tau}$ le taux d'actualisation réel ${ }^{(12)}$ pour une échéance à $\tau$ périodes, avec $i_{0}=1$. Les espérances mathématiques $E_{t}($.$) sont prises$ conditionnellement à l'information disponible au début de la période t. Le prix du capital se fixe à l'équilibre au point qui assure l'égalité suivante :

(2) $\frac{P_{k t}}{P_{t}}=\sum_{\tau=0}^{\infty} \frac{\delta_{\tau} E_{t}\left(q_{t+\tau} A_{t+\tau}\right)}{\left(1+i_{\tau}\right)^{\tau}}$

L'équation (2) désigne le modèle d'actualisation des profits. Elle établit la valeur d'équilibre du prix du capital en fonction du flux anticipé de profit, qui dépend du taux de marge et de la productivité apparente du capital. En quoi ce modèle d'actualisation des profits se distingue-t-il des modèles d'actualisation des dividendes et des bénéfices? Pour le comprendre, écrivons les trois modèles sous forme d'une équation de récurrence d'ordre un. Pour simplifier, normalisons le prix de la valeur ajoutée : $P_{t}=1$, et supposons l'absence d'inflation. L'équation (2) s'écrit sous la forme de l'équation de récurrence suivante :

(3) $P_{k t}=q_{t} A_{t}+\frac{\delta_{1} E_{t}\left(P_{k t+1}\right)}{1+i_{1}}$

Une unité de capital engagée dans la production permet d'engendrer le montant de valeur ajoutée $A_{t}$, partagée entre salaires et profits. Le profit ${ }^{(13)}$ est défini en fonction du taux de marge et vaut $q_{t} A_{t}$. Compte tenu de l'usure et de l'obsolescence de l'unité de capital disponible en $\mathrm{t}$, il restera la quantité résiduelle $\delta_{l}$. Une unité de capital disponible en $\mathrm{t}$, de prix $P_{k t}$, donne ainsi droit au profit $q_{t} A_{t}$, et pourra être revendue $\delta_{1} P_{k t+1}$. Si on note $i_{1}$ le taux d'actualisation à échéance d'une période, on observe que le prix du capital est bien défini par l'équation (2), après actualisation de la valeur résiduelle future du capital.

Comparons à présent ce modèle avec le modèle d'actualisation des dividendes. Partant du profit $q_{t} A_{t}$ dégagé au cours de la période d'exploitation, une partie est distribuée en dividendes. Notons $d_{t}$ le taux de dividende, de sorte que le dividende versé vaut $d_{t} q_{t} A_{t}$. Dans le modèle d'actualisation des dividendes, seul ce terme est valorisé en première période, le reste du profit étant valorisé à travers le capital où il est réinvesti. En effet, la partie non distribuée $\left(1-d_{t}\right) q_{t} A_{t}$ sert à racheter des biens d'investissement. Notons $P_{I t}$ le prix d'un bien d'investissement. Pour simplifier, supposons que ce prix est fixé contractuellement à l'avance, de sorte que $P_{I t+1}$ est connu en t. Faisons l'hypothèse simple suivante quant à la technologie de transformation de l'investissement en capital : une unité de bien d'investissement est transformée sans coût en une unité de capital. La partie non distribuée du profit permet alors d'accroître le capital de la quantité $\left(1-d_{t}\right) q_{t} A_{t} / P_{I t+1}$. Ainsi, une unité de capital donne droit à la perception d'un dividende $d_{t} q_{t} A_{t}$, et confère aussi un droit de propriété sur le montant de capital suivant, formé de l'unité de capital résiduelle, et du capital acheté avec les profits non distribués : $\delta_{1} P_{k t+1}+\left(1-d_{t}\right) q_{t} A_{t} P_{k t+1} / P_{I t+1}$. Ce montant anticipé est actualisé, et ajouté au dividende perçu, pour former le prix du capital :

(4) $P_{k t}=d_{t} q_{t} A_{t}$

$+\frac{\delta_{1} E_{t}\left(P_{k t+1}\right)}{1+i_{1}}+\frac{\left(1-d_{t}\right) q_{t} A_{t} E_{t}\left(P_{k t+1}\right) / P_{I t+1}}{1+i_{1}}$

Avant de discuter de la comparaison de ces modèles, présentons le troisième modèle, celui d'actualisation des bénéfices. La valorisation par le bénéfice se situe à mi-chemin entre la valorisation par le profit et la valorisation par le dividende. Le profit donne lieu à un bénéfice, c'est-à-dire la part du profit qui reste après remplacement du capital consommé. La consommation de capital fixe est égale à $1-\delta_{1}$. Cette consommation donne lieu à une dotation aux amortissements, de valeur $\left(1-\delta_{1}\right) P_{I t+1}$, qui sert à racheter des biens d'investissement pour maintenir le capital au volume unitaire d'origine. Le bénéfice est égal au profit dont on a retranché cette dotation aux amortissements, soit $q_{t} A_{t}-\left(1-\delta_{1}\right) P_{I t+1}$. Une unité de capital disponible en $\mathrm{t}$ donne droit à ce bénéfice, et sera toujours entièrement disponible à la période suivante, puisque la dépréciation a été amortie. Il en résulte, après actualisation de la valeur future anticipée de l'unité de capital, que le modèle d'actualisation des bénéfices s'écrit sous la forme :

(5) $P_{k t}=q_{t} A_{t}-\left(1-\delta_{1}\right) P_{I t+1}+\frac{E_{t}\left(P_{k t+1}\right)}{1+i_{1}}$ 
Les équations (3), (4) et (5) sont distinctes. Pour bien en comprendre les différences, soulignons la condition qui les rend toutes trois équivalentes : $E_{t}\left(P_{k t+1}\right)=P_{I t+1}\left(1+i_{1}\right)$. Sous cette condition, les équations (4) et (5) peuvent être simplifiées par $P_{I t+1}$, et s'écrivent sous la forme (3). Cette condition définit l'existence d'une zone de non arbitrage. Lorsque le prix futur anticipé du capital est égal à $P_{I t+1}\left(1+i_{1}\right)$, les propriétaires du capital ne tirent aucun surprofit supplémentaire liée à la transformation des biens d'investissement en capital et à leur revente sur le marché du capital. Si le prix futur anticipé du capital est supérieur à cette valeur seuil, l'investissement et la revente du capital installé est profitable. Ce surprofit anticipé n'est pas du tout pris en compte dans le modèle d'actualisation des profits, l'évaluation des profits ne tenant pas compte de la stratégie de la firme ; par contre il l'est pleinement dans le modèle d'actualisation des dividendes, et partiellement dans le modèle d'actualisation des bénéfices. Il appert que les trois modèles peuvent produire des évaluations différentes, parce qu'ils ne tiennent pas compte de la même information. Le modèle d'actualisation des profits évalue le capital sans tenir compte du partage du profit entre dividendes et réinvestissement. Le modèle d'actualisation des bénéfices évalue le capital après amortissement, sans anticipation de ce qui sera fait du bénéfice. Le modèle d'actualisation des dividendes repose sur l'anticipation de la stratégie de la firme, de l'utilisation qui sera faite des profits, de leur possible réinvestissement.

D'un point de vue normatif, l'utilisation du modèle d'actualisation des profits est préférable, car il repose sur l'hypothèse que le capital est évalué à sa juste valeur relativement au bien d'investissement. Il serait en effet paradoxal de proposer une évaluation normative fondée sur l'anticipation de surprofits liés à l'opportunité d'acheter des biens d'investissement à prix avantageux.

D'un point de vue opérationnel, le modèle d'actualisation des profits est aussi le plus simple, et celui des dividendes le plus complexe. En effet, l'accumulation du capital produit des dividendes croissants, ce qui signifie que la prise en compte d'un futur lointain ne décroît que lentement. La formule d'actualisation des dividendes dépend fortement de l'anticipation de variables très éloignées dans le temps. Ce modèle est alors soumis à un risque d'estimation statistique assez fort. Le modèle d'actualisation des bénéfices est déjà un peu moins sensible à ce problème, le stock de capital pris en compte dans l'évaluation étant constant. Le flux de bénéfices futurs est alors un flux décroissant après prise en compte de l'actualisation. Mais la décroissance des bénéfices actualisés est assez lente, et l'estimation du modèle reste influencée par l'incertitude statistique qui pèse sur les prévisions de bénéfices à des échéances assez lointaines. Le modèle le plus simple est bien le modèle d'actualisation des profits, le capital pris en compte dans l'évaluation étant décroissant en raison de l'obsolescence et de l'usure. Le flux de profits décroît alors rapidement, compte tenu de l'actualisation et de l'obsolescence. Le prix du capital dépend d'anticipations de profits à échéances relativement courtes, les profits à échéance lointaine n'ayant que peu d'influence.

Ces deux raisons nous amènent à préférer le modèle d'actualisation des profits pour offrir une évaluation normative du prix du capital. Ecrit sous la forme générale de l'équation (2), le modèle n'est cependant n'est guère opérationnel, car il possède une suite infinie de termes $E_{t}\left(q_{t+\tau} A_{t+\tau}\right)$ non paramétrés. Pour être rendue utilisable dans la pratique, l'équation (2) doit être paramétrée. La solution que nous adoptons consiste à paramétrer statistiquement le terme $E_{t}\left(q_{t+\tau} A_{t+\tau}\right)$, en utilisant un modèle de prévision des valeurs futures de $q_{t} A_{t}$. Comme nous l'avons déjà souligné, les variables à prévoir sont relativement stables dans la longue période, et leur influence sur le prix décroît avec l'échéance. L'inertie statistique des variables fondamentales, décrite dans la première section, permet de recourir à des hypothèses paramétriques simples. Si on suppose que la variable produit $q_{t} A_{t}$ est markovienne, toutes les espérances du type $E_{t}\left(q_{t+\tau} A_{t+\tau}\right)$ sont des fonctions linéaires de $q_{t} A_{t}$. Il existe alors des paramètres positifs ${ }^{(14)} \alpha$ et $\beta$, fonctions des paramètres de ce processus markovien, mais aussi fonctions des taux de dépréciation et d'actualisation, tels que le prix du capital puisse s'écrire tout simplement :

(6) $\frac{P_{k t}}{P_{t}}=\alpha+\beta q_{t} A_{t}, \alpha \geq 0, \beta>0$

L'équation (6), plus parcimonieuse que l'équation (2), mais dépendant d'une hypothèse simplificatrice, établit que le prix réel du capital est simplement défini comme une fonction linéaire du profit (réel) courant. La forme de cette équation a par ailleurs l'avantage d'être insensible 
à des changements de mesure du capital et de la valeur ajoutée. Car bien évidemment, la série de prix $P_{k t}$ n'est pas observable dans la réalité, et on observe seulement à la place de celle-ci une série indicielle du prix des actions. La même remarque peut être faite pour la série de prix $P_{t}$. Chaque indice de prix étant cependant proportionnel au prix d'une unité, l'équation (6) reste valide même lorsque $P_{k t}$ et $P_{t}$ représentent de tels indices. Seules les valeurs des paramètres $\alpha$ et $\beta$ sont affectées par ce remplacement. C'est sous la forme de l'équation (6) que nous estimons par la suite le modèle d'actualisation des profits.

\section{Tendance et cycles longs du prix des actions}

Lorsque les fondamentaux économiques varient, le prix du capital varie. Mais comme ces fondamentaux sont stables dans la longue période, le prix réel du capital possède aussi une valeur de longue période, qui devrait jouer le rôle d'un phare pour les investisseurs. Au sens marshallien du concept, ce prix peut être appelé prix (réel) naturel du capital. Si l'on pose que $q_{t}$ et $A_{t}$ sont égaux à leurs valeurs de longue période notées respectivement $\mathrm{q}$ et $\mathrm{A}$, alors il découle de l'équation (2) que le prix réel naturel du capital, noté $P_{k} / P$, vaut :

(7) $\frac{P_{k}}{P}=\gamma q A$, avec $\gamma=\sum_{\tau=0}^{\infty} \frac{\delta_{\tau}}{\left(1+i_{\tau}\right)^{\tau}}$

De même que la stabilité dans la longue période du taux de marge et de la productivité apparente du capital ont conféré à leurs valeurs un statut de «constantes de la macroéconomie », la stabilité dans la longue période du prix réel $\mathrm{du}$ capital devrait aussi offrir aux investisseurs et observateurs de la sphère financière une autre constante de longue période de la macroéconomie, le prix naturel du capital.

Compte tenu de la stationnarité (en espérance) des variables stochastiques $q_{t}$ et $A_{t}$, l'espérance de ces variables est égale à leur valeur de longue période, de sorte que l'espérance du prix réel du capital est aussi égale à sa propre valeur de longue période: $\quad E\left(P_{k t} / P_{t}\right)=P_{k} / P=\gamma q A$. Le prix naturel du capital peut tout simplement être estimé par la moyenne d'une chronique longue du rapport $P_{k t} / P_{t}$. La série $P_{k t} / P_{t}$ évolue autour d'un niveau de longue période, qui sert de point de référence, les variations autour de cette constante étant expliquées par les seules variations des fondamentaux par rapport à leurs propres valeurs de longue période.

La figure 4, présentée ci-après, trace les variations du prix réel du capital pour le cas du marché français, sur presque quatre-vingt-dix ans ${ }^{(15)}$. La première question à se poser, au vu de cette figure, est celle de l'existence d'un niveau de longue période constant du prix réel du capital. $\mathrm{La}$ série visible sur ce graphique est-elle compatible avec l'hypothèse d'une espérance (totale) constante? Apparemment, la série est très variable. Mais ces variations sont-elles uniquement le fait de la volatilité ou sont-elles dues aussi à l'existence d'un trend? L'observation de la figure 4, tout arbitraire qu'elle soit, ne laisse deviner aucune tendance haussière ou baissière manifeste. On peut croire à l'hypothèse de constance de l'espérance de la série, et à l'existence d'un prix (réel) naturel du capital.

\section{Figure 4 : le prix réel des actions françaises} (1920-2008)

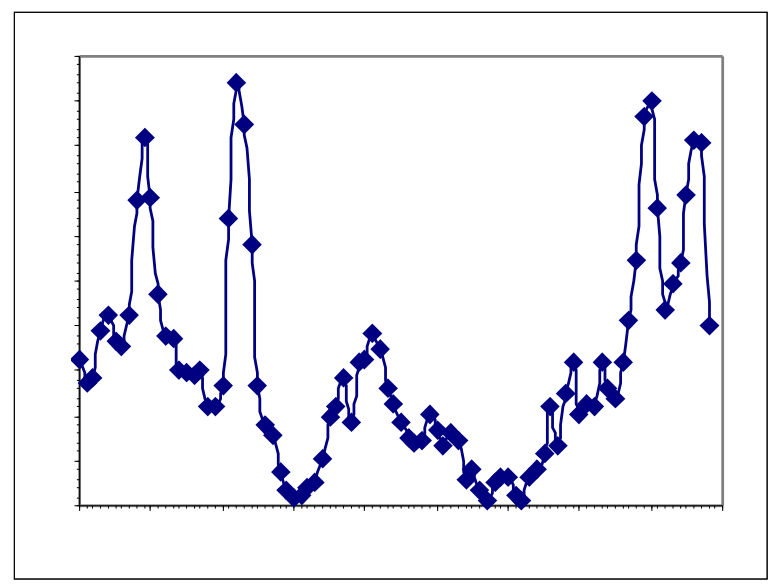

Chronique de 1920 à 2008 d'un indice du prix des actions françaises/ prix de la valeur ajoutée en France

L'existence de l'espérance de la série n'est cependant pas compatible qu'avec la seule hypothèse de stationnarité (au sens large) du processus. Un processus intégré d'ordre 1, sans dérive, possède une espérance constante. Un tel processus, dont la marche aléatoire sans dérive est l'exemple le plus connu, est par contre non stationnaire en variance. D'un point de vue statistique, il se peut que la série visible sur la figure 4 soit non stationnaire en variance. Pourtant, la différence entre les deux modèles est cruciale. Dans un modèle stationnaire au second ordre, l'espérance mais aussi la variance existent 
et sont finies. La probabilité que le processus s'écarte de son espérance est bornée, et la série ne peut diverger. Dans un modèle stationnaire seulement au premier ordre, la série diverge et peut s'éloigner indéfiniment de son espérance. Si les profits jouent effectivement comme une force de rappel, le prix réel du capital ne devrait pas s'éloigner durablement du prix naturel, restant toujours dans un voisinage plus ou moins étendu autour de celui-ci.

Pour qui est coutumier de l'analyse chronologique, il est clair que la série visible sur la figure 4 présente un comportement qui n'est pas éloigné de celui d'un processus (sans dérive) intégré d'ordre 1. Une marche aléatoire sans terme constant pourrait produire une série à l'évolution décrite sur ce graphique. L'hypothèse de stationnarité au second ordre de la série doit-elle cependant être rejetée ? Nous avons procédé aux tests de Phillips-Perron (Phillips et Perron, 1988) de l'existence d'une racine unitaire. Les résultats, relatifs aux statistiques de $\operatorname{test}^{(16)} \hat{z}_{\alpha}$ et $\hat{z}_{t}$, indiquent des probabilités critiques égales respectivement à $3,19 \%$ et $6,46 \%$. La crédibilité associée à l'hypothèse nulle d'une racine unitaire est assez faible ${ }^{(17)}$. Au regard de ces valeurs, l'hypothèse de stationnarité au second ordre ne doit pas être remise en cause.

Ayant admis que le processus est stationnaire, l'application de la méthodologie de Box-Jenkins (lecture des corrélogrammes, estimation et vérification) conduit à identifier et estimer le processus linéaire compatible avec les données, un autorégressif d'ordre 3 :

$$
\begin{aligned}
& \left(p_{t}-0,402\right)=1,45\left(p_{t-1}-0,402\right)-0,87\left(p_{t-2}-0,402\right) \\
& +0,26\left(p_{t-3}-0,402\right)+\hat{\varepsilon}_{t}
\end{aligned}
$$

La forme du processus n'est ici qu'anecdotique. Ce qui importe est la valeur estimée ${ }^{(18)}$ du niveau de longue période, l'espérance du processus: 0,402. Cette valeur est cruciale pour les investisseurs, car elle définit la norme d'évaluation du prix des actions dans la longue période. Il apparaît cependant que cette estimation contient une grande incertitude, puisqu'un intervalle de confiance au niveau 0,95 donne la réalisation numérique suivante (sous approximation asymptotique gaussienne) : $[0,278 ; 0,526]$. L'estimation varie ainsi quasiment du simple au double!! Ainsi, la constante de longue période, paramètre de référence si l'on en croît le modèle théorique, n'est pas connue précisément, et même plus que cela, elle n'est estimée que très grossièrement, ce qui lui fait perdre son caractère opérationnel. Presque un siècle d'observations ne suffit pas à estimer précisément sa valeur.

L'imprécision relative à cette constante met dans une position inconfortable qui veut procéder à une évaluation objective du prix du capital. Mettonsnous ainsi à la place d'un investisseur à la fin de l'année 2008, année marquée par une forte baisse des cours boursiers, en France comme dans le reste du monde. A la fin de cette année, l'indice réel du prix des actions est tombé à 0,501 . Mais cette valeur reste nettement supérieure à la valeur de longue période estimée, 0,402 . Un indice de 0,501 n'est soutenable que si les fondamentaux sont eux-mêmes supérieurs à leurs valeurs de longue période. Dans le cas contraire, on conclut au fait que les actions sont surévaluées de $20 \%$ en termes réels, ce qui appelle pour corriger ce déséquilibre à une prolongation du mouvement baissier du marché. Pour faire cette comparaison, nous avons cependant utilisé une estimation du prix naturel dont le moins qu'on puisse dire est qu'elle est très incertaine. Si nous avions raisonné avec la valeur plancher de notre intervalle de confiance, soit 0,278 , le déséquilibre serait apparu avec plus d'importance, et aurait nécessité pour correction une baisse de presque $50 \%$ du prix réel des actions! Si au contraire nous avions raisonné avec la valeur plafond de l'intervalle d'estimation, soit 0,526 , nous aurions conclu au contraire que le prix réel des actions est légèrement inférieur à sa valeur de longue période! On le voit, l'imprécision relative à la valeur de longue période du prix du capital l'empêche de jouer son rôle de phare. Et sans la connaissance de la valeur précise de ce paramètre, le modèle d'actualisation des profits se trouve soumis à une incertitude statistique qui empêche de l'utiliser objectivement.

A cette première conclusion, il faut en ajouter une seconde, relative à l'observation de variations cycliques relativement longues. On perçoit très bien sur la figure 4 l'existence de trois cycles, le premier se terminant au début des années 50 et le deuxième se terminant au début des années 80 . Ces cycles ne sont pas réguliers et durent plusieurs décennies, celui de la période actuelle ayant commencé il y a presque 30 ans et n'étant pas encore terminé. On reconnaît clairement les épisodes spéculatifs liés à la crise de 1929, à la bulle technologique de 2000 , et à la crise financière actuelle. Un autre pic apparait en 1942. Celui-ci n'est cependant pas caractéristique d'un épisode spéculatif financier, mais est le résultat d'un artefact statistique ${ }^{(19)}$. 
L'existence de ces cycles financiers longs constitue un élément très important, et il est crucial de ne pas se tromper en pointant du doigt de telles fluctuations cycliques. Ces cycles existent-ils réellement, ou ne sont-ils qu'un artefact statistique liés à quelques épisodes spéculatifs intenses qui tirent vers le haut, par endroits, la série de prix relatif, créant ainsi l'illusion de ces amples fluctuations?

Comme nous le montrons ci-après, ces cycles existent bel et bien, et cela indépendamment (d'un point de vue statistique) des pics spéculatifs. Nous les faisons apparaître grâce à une procédure de lissage robuste, qui veille à ce que la série lissée ne soit pas «tirée» par les bulles, et que ses variations ne soient pas un artefact statistique du à la présence de quelques points aberrants. $\mathrm{La}$ robustesse de la méthode utilisée prévient ce risque, et permet le constat de l'existence de ces cycles. Dans une première étape, nous avons tronqué la distribution empirique des prix. Pour éliminer l'influence des bulles, nous avons réduit la valeur des $15 \%$ d'observations les plus élevées à la valeur du $85^{\text {ème }}$ centile (soit 0,683 ). De cette façon, les pics visibles sur la figure 4 disparaissent, et nous sommes sûrs que l'apparition de cycles, à partir de cette série tronquée, n'est pas la conséquence de quelque outlier. Dans une seconde étape, nous avons procédé à une régression non paramétrique robuste de $\mathrm{p}$ sur $\mathrm{t}$ en utilisant la méthode loess ${ }^{(20)}$ de Cleveland (1979), pour obtenir un lissage robuste de la série du prix réel des actions (figure $5)$.

Figure 5 : série du prix réel des actions et série lissée

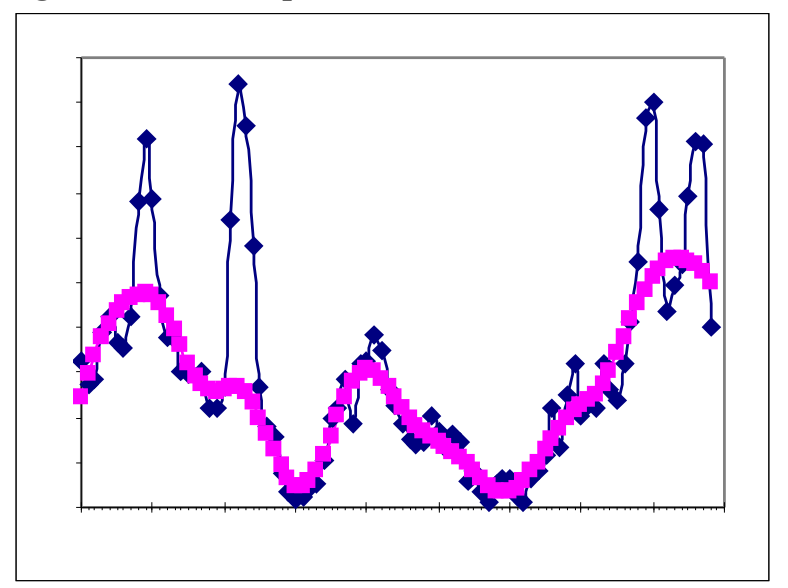

On distingue très clairement sur le graphique une succession de trois cycles de durées et d'amplitudes inégales. Les sommets de ces cycles coïncident à chaque fois avec une bulle spéculative, qui n'apparaît que comme la partie émergée d'un iceberg, qui trouve ses racines dans un processus long de hausse des prix qui commence dix à vingt ans auparavant. L'explication de ces cycles est cruciale pour la compréhension du mouvement long du prix réel du capital. Ces cycles sont-ils la manifestation de cycles fondamentaux, c'est-à-dire de variations cycliques du profit réel, ou sont-ils la résultante d'un cycle du crédit qui expliquerait la majeure partie des variations du prix des actions, indépendamment de l'influence des fondamentaux ?

\section{Le prix réel des actions dans la courte période : les profits n'expliquent pas tout}

Si les variables fondamentales s'écartent de leur niveau de longue période, le prix réel du capital s'éloigne du prix naturel. L'existence de variations cycliques du taux de marge et de la productivité du capital constitue une explication plausible des cycles visibles sur la figure 4. Si l'on centre l'équation (6) sur la valeur de longue période du prix du capital: $P_{k} / P=\alpha+\beta q A$, alors l'écart du prix réel du capital par rapport au prix naturel vaut :

(8) $\frac{P_{k t}}{P_{t}}-\frac{P_{k}}{P}=\beta\left[q_{t} A_{t}-q A\right]$

Tout écart du profit courant par rapport à sa valeur de long terme $q A$ se répercute dans le prix du capital, qui s'écarte alors du prix naturel. De telles variations des fondamentaux sont-elles réellement à l'origine des variations observées du prix du capital ? Sur la figure 4, on voit que le prix réel du capital a connu trois phases de hausse que l'on peut résumer approximativement: 1920-1930, 1950-1960, 1980-2000. Les périodes intermédiaires correspondent à des phases de baisse. Si le prix du capital est fixé, ne serait-ce qu'approximativement, selon le modèle d'actualisation (2), alors ces périodes devraient aussi correspondre à des périodes de hausse du profit.

Prigent (1999), Cette et Sylvain (2001), Timbeau (2002), Sylvain (2007) confirment que sur les 35 dernières années, le taux de marge a évolué dans un sens conforme aux variations du prix du capital. Le taux de marge a ainsi plus ou moins fortement baissé (selon les estimations) dans les années 70 , faisant baisser les profits, avant de se 
redresser durant les vingt années suivantes. La politique économique française menée depuis le début des années 80 , plus favorable aux détenteurs des capitaux, a permis de rétablir le taux de marge à un niveau plus rémunérateur pour eux, et sans doute faut-il y voir une explication partielle des variations du prix des actions à la hausse. Les années 60 furent quant à elles marquées par une relative stabilité de la norme de partage de la valeur ajoutée. Il semble qu'il ne faille pas imputer au conflit salariés/capitalistes le processus de baisse du prix des actions des années 60 . Prigent (1999) précise cependant qu'à la fin des années 90, le taux de marge avait retrouvé son niveau de la fin des années 50. Pourtant, durant la même période, le prix réel du capital a quasiment doublé ! L'évolution du taux de marge ne peut à lui seul expliquer les énormes variations du prix des actions.

L'autre variable composant le profit, la productivité du capital, compense-t-elle les insuffisances de la première ? Le rapprochement des figures 2 et 5 conduit à douter que la productivité du capital puisse jouer un rôle explicatif important dans l'explication des variations du prix du capital. Il est vrai que la productivité du capital a baissé durant la décennie 70 , alors que le prix réel des actions était luimême à la baisse, ce qui va dans le sens du modèle, mais elle a aussi baissé durant les quinze années suivantes alors que le prix des actions remontait, et elle diminuait encore récemment entre 2000 et 2005 , alors que le prix réel du capital continuait d'augmenter.

Pour approfondir l'analyse empirique du modèle, une analyse économétrique semble nécessaire. L'équation (6), qui stipule que le prix réel du capital est une fonction linéaire du profit courant, peut en effet faire l'objet d'une telle analyse. Dans le plan $\left(q_{t} A_{t}, P_{k t} / P_{t}\right)$, un nuage de points échantillonnés de ces deux variables devrait s'étirer parfaitement le long d'une droite de pente $\beta$ et d'ordonnée à l'origine $\alpha$. Le $R^{2}$ de cette régression a une signification particulière, puisqu'il désigne le pourcentage des variations du prix du capital qui est expliqué par les fondamentaux. Par exemple, un $R^{2}$ de 0,60 serait le signe que $60 \%$ des variations des prix sont expliquées par les variations des profits, le reste des variations étant expliquée par l'existence de forces déséquilibrantes, la plus importante étant certainement une expansion inadaptée du crédit et de l'endettement.
Compte tenu des données disponibles, nous avons procédé à l'estimation du modèle sur la période 1970-2002. Les données de productivité et de taux de marge utilisées sont celles présentées dans les figures 2 et 3 . Nous avons calculé le profit $q_{t} A_{t}$, et avons régressé sur la période 1970-2002 notre série de prix réel du capital sur cette variable. La droite de régression tirée de l'équation (6) est représentée sur la figure 6 .

Figure 6 : régression du prix réel des actions françaises sur le profit réel du capital

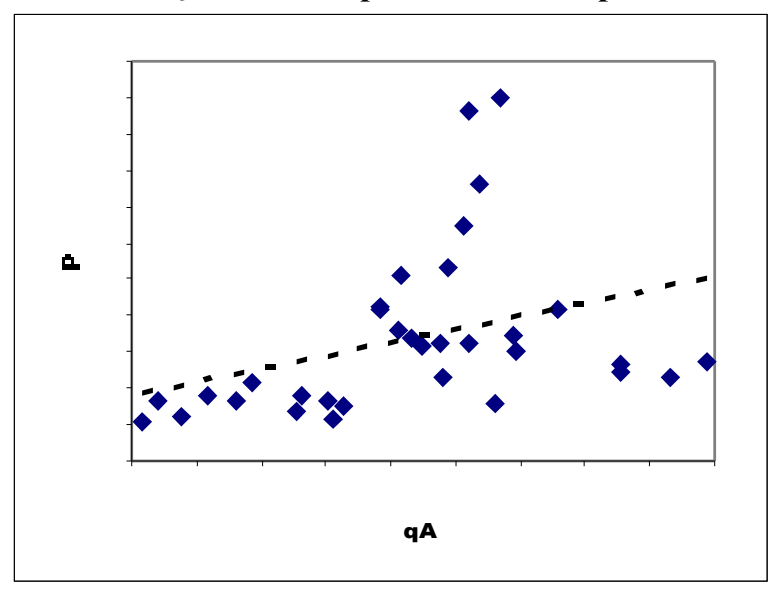

Le modèle estimé, $\hat{P}_{k t} / P_{t}=-0,228+1,845 q_{t} A_{t}$, présente un $R^{2}$ assez faible $(0,127)$, indiquant que l'essentiel de l'explication des variations de prix réel des actions se trouve ailleurs que dans les variations des fondamentaux. Si le terme constant ne semble pas significativement différent de 0 , la pente du modèle paraît au contraire significative ${ }^{(21)}$. Le prix réel des actions ne semble pas détaché des profits, mais le lien semble assez lâche. Cette faiblesse de l'explication financière par les fondamentaux peut sans doute être corrigée en partie par une amélioration du modèle d'anticipation des profits. Sans doute l'hypothèse markovienne est-elle trop forte. Cependant, des tests économétriques effectués montrent que la variable retardée $q_{t-1} A_{t-1}$, si on l'ajoute comme régresseur supplémentaire, n'est pas significative. La solution n'est donc pas d' «augmenter » le processus. Une solution plus efficace serait de trouver les variables dont disposent les investisseurs dans leur ensemble informationnel et qui leur sert à prévoir les profits futurs. Sans doute pourrait-on améliorer de la sorte le modèle d'anticipation des fondamentaux ${ }^{(22)}$.

Cela dit, il faut rappeler que la variable explicative peut faire l'objet d'importantes erreurs de mesure. C'est vrai pour les deux composantes, mais peut-être plus encore pour la productivité du capital. Comme nous l'avons déjà souligné, le stock de capital est estimé, et non pas observé, et 
cette estimation est très sensible aux hypothèses faites sur la durée de vie des divers équipements. Il se peut que les variations de la productivité observables sur la figure 2 soient en grande partie le fait d'erreurs de mesure. Si, comme ont pu l'écrire certains auteurs, on peut supposer que la productivité a été, après le premier choc pétrolier, essentiellement stable autour d'une constante de moyen terme, alors le modèle peut être simplifié. Si on pose que $A_{t}=A \forall t$, l'équation (6) devient :

(9) $\frac{P_{k t}}{P_{t}}=\alpha+\gamma q_{t}, \gamma=\beta A$

Les variations du prix réel des actions s'expliquent alors uniquement par celles du taux de marge. Nous avons procédé à l'estimation de cette équation (figure 7).

Figure 7 : régression du prix réel des actions françaises sur le taux de marge

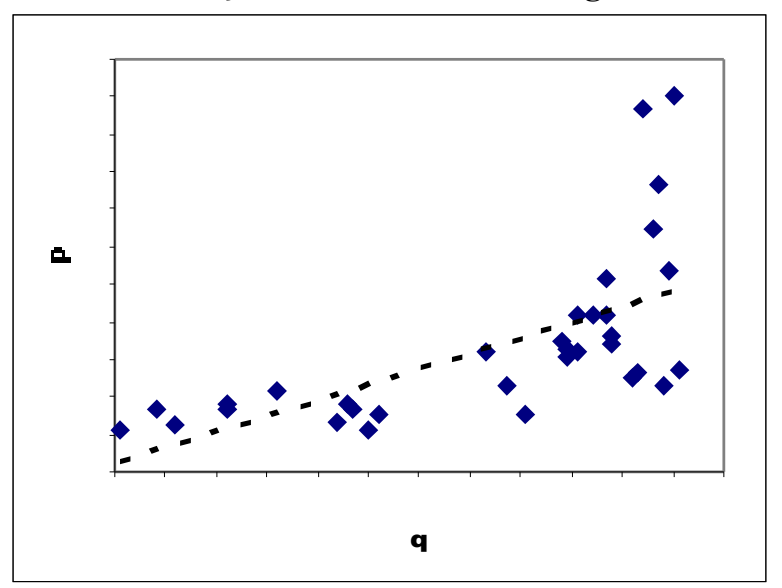

Le modèle estimé, $\hat{P}_{k t} / P_{t}=-0,854+4,188 q_{t}$, présente un $R^{2}$ bien supérieur à celui du premier modèle $(0,40)$. Le taux de marge explique une partie des variations du prix réel des actions françaises $^{(23)}$, bien mieux «en solitaire » que lorsqu'on le combine à la productivité du capital. Cela dit, même si l'ajustement du modèle aux données est largement amélioré, il reste très éloigné de cet ajustement parfait que suppose le modèle. La majeure partie des variations du prix réel des actions n'est pas le fait de celles du profit. $\mathrm{Si}$ les variations des fondamentaux ne peuvent expliquer celles du prix réel des actions, l'explication des cycles du prix des actifs financiers doit être recherchée dans une théorie du cycle du crédit (Aglietta, 2008). C'est parce que l'expansion du crédit est beaucoup trop rapide que les actifs financiers (et autres actifs patrimoniaux) s'apprécient en valeur réelle beaucoup plus qu'ils ne devraient. Un excès de crédit provoque une sur-appréciation tendancielle des actifs financiers.
Ces cycles ne sont cependant pas des cycles courts, comme le montre clairement la figure 5 . Même s'il peut y avoir des cycles dans le cycle, l'excès de crédit et d'endettement est un processus long qui prend visiblement plusieurs décennies, et dont les bulles spéculatives ne semblent être que des manifestations tardives. Comme on le voit sur la figure 5, les bulles apparaissent au sommet des cycles, et il est sans doute déjà trop tard pour corriger la trajectoire en douceur lorsque la partie émergée de l'iceberg apparaît.

\section{Conclusion}

Des cycles financiers longs, s'étalant sur plusieurs décennies, caractérisent l'évolution du prix des actions françaises. Contrairement à ce que suggèrerait une théorie générale de l'équilibre des marchés, il semble difficile de cataloguer ces cycles financiers dans la catégorie des variations cycliques d'équilibre. Les variables fondamentales ont un effet significatif incontestable sur le cycle, mais leurs fluctuations n'expliquent pas la moitié des variations du prix réel du capital. Les marchés financiers sont soumis à des mécanismes déstabilisateurs puissants, qui expliquent que l'expansion du crédit et l'endettement peuvent continuer d'alimenter la hausse des cours, alors même que le marché est au-delà de son niveau d'équilibre de longue période. La question se pose de savoir ce qui peut permettre que d'importants déséquilibres subsistent sur des périodes qui se mesurent en décennies. Les agents sont-ils irrationnels à ce point, cédant à l'euphorie en certaines périodes, à la panique en d'autres, en tout cas versant continuellement dans l'exagération? Ou existe-til des mécanismes déstabilisant qui produisent une volatilité excessive en dépit de la rationalité individuelle des agents?

Répondre par l'affirmative à la seconde question paraît ouvrir des portes quand la même réponse à la première paraît au contraire les fermer. Si l'on suppose que l'excès de volatilité du prix des actifs est le produit de la versatilité irrationnelle des agents, une action correctrice de la banque centrale peut être bénéfique. En agissant sur les marchés (par des variations de taux, des injections ou retraits de liquidités) ou en contrôlant les anticipations des agents par des déclarations sur ses intentions, la banque centrale peut influer sur la trajectoire des prix et la ramener vers son sentier d'équilibre fondamental. Dans cette 
situation, la cause des distorsions est aussi son symptôme, et un traitement "symptomatique » suffit à rétablir le principe d'une juste valorisation des actifs.

$\mathrm{Si}$ au contraire ce sont des mécanismes déséquilibrants qui produisent ces écarts durables des prix à leurs valeurs fondamentales, et cela en dépit d'un comportement rationnel des agents, alors l'interventionnisme du banquier central risque d'être vain. Toute action correctrice risque de ne produire que des effets temporaires. Le traitement symptomatique peut être inefficace, si la cause des distorsions subsiste.

Est-il crédible d'attribuer à la rationalité imparfaite des agents l'existence de cycles dont la durée se mesure en décennies? Que les agents puissent se tromper ou présenter des travers de comportement est un fait. Leur surréaction ou sous-réaction aux informations occasionnent un excès de volatilité des cours boursiers. La finance comportementale s'est ainsi développée pour offrir une meilleure compréhension des comportements financiers. Mais il ne faut sans doute pas attribuer aux comportements des agents toute l'explication du phénomène. L'instabilité financière est principalement liée au crédit et à l'endettement (Borio et Lowe, 2002), et il ne faut pas attendre d'une politique monétaire menée à destination des marchés financiers qu'elle corrige le prix des actifs, si par ailleurs le crédit et l'endettement ne sont pas ramenés à des niveaux compatibles avec l'équilibre de l'économie. Cependant, cela ne signifie pas que la banque centrale doit rester en-dehors des marchés, et prendre acte de variations de prix qu'elle pourrait juger inexplicables au regard des fondamentaux. Son action, sous la forme d'un simple monitoring des marchés financiers, serait la bienvenue. Compte tenu de la masse phénoménale des travaux statistiques produits par les économistes des institutions monétaires et financières, nationales et internationales, il est étonnant de constater la faible importance de ceux consacrés à l'évaluation financière normative. Il ne paraît pas impossible de faire publier régulièrement par des experts accrédités des indicateurs sur l'écart du marché à un prix de référence. De telles informations, si elles étaient jugées crédibles par le marché, auraient l'avantage de faire converger les anticipations, et sans doute de réduire un peu cette excessive volatilité du prix des actifs.

\section{Notes}

(1) C'est par exemple le modèle que Keynes (1936) utilise pour définir la notion d'efficacité marginale du capital. Il est à noter que Keynes parle de « rendement» plutôt que de "profit», mais les deux termes peuvent être utilisés, le rendement étant aujourd'hui défini par les comptables nationaux comme le profit obtenu à l'aide d'une unité de capital.

(2) Nous utilisons l'expression de longue période en référence à son acception statistique, c'est-à-dire lorsque nous cherchons à expliquer le trend du prix. L'analyse de courte période fait référence à l'explication des variations autour de la tendance.

(3) L'approche à un niveau global permet une importante simplification, couramment posée dans les modèles DGSE qui intègrent un secteur financier (voir la synthèse de Beaubrun-Diant et Matheron, 2008), et que nous avons reprise. Au niveau d'une économie globale, l'endettement peut être négligé, en ce sens que la somme des prêts est égale à la somme des emprunts. Cette simplification permet de confondre valeur des actifs et valeur des fonds propres.

(4) Ce flux est décroissant dans la mesure où la dépréciation du capital est plus importante qu'une éventuelle hausse de la productivité du capital, ce qui est toujours le cas dans la réalité.

(5) C'est la définition que Keynes (1936) donne du prix d'offre du capital tout au début du chapitre 11 de sa Théorie Générale (sauf qu'il raisonne en termes nominaux et non réels).

(6) On pourrait ainsi définir un prix éthique du capital.

(7) Pour faire le calcul de $Y / K$, nous avons utilisé dans la base de données du Cepii (disponible à l'adresse www.cepii.fr/francgraph/bdd/villa/mode.htm) les séries PIBZQ et KZE pour représenter respectivement $Y$ et $K$. La première série (du fichier " prod») désigne le PIB en volume, calculé par la production, aux prix de 1938. La seconde série (du fichier "long») désigne le stock de capital brut (matériel et bâtiment des entreprises, dommages de guerre compris), aux prix de 1938 .

(8) Les données de valeur ajoutée et de formation brute de capital fixe (hors dépenses de logement) sont mesurées en volume aux prix de 2000. Les données de VA, comme de FBCF, ne concernent que le seul secteur institutionnel des sociétés et entreprises individuelles non financières. Source: Banque de Données Macro-économiques de l'Insee,

www.bdm.insee.fr/bdm2.

(9) Nous avons suivi Sylvain (2001) pour le choix d'une loi de mortalité soudaine. La durée de vie des équipements est de 12 ans en moyenne (Cette et Szpiro, 1988). Sylvain (2001) montre que les hypothèses relatives à la durée de vie des équipements jouent peu sur le diagnostic «qualitatif» de la productivité. Ainsi, l'estimation du stock de capital en utilisant une mortalité différente change les valeurs de 
notre série, mais ne change pas vraiment son évolution dans le temps. Cela permet d'expliquer la différence de productivité observable sur les graphiques 1 et 2 , pour la période commune 1970-1985, que l'on doit imputer au fait que les sources des données ne sont pas les mêmes, mais aussi au fait que les hypothèses relatives à la durée de vie des équipements sont différentes d'une estimation à l'autre.

(10) La prise en compte d'une correction de la nonsalarisation est notamment un élément méthodologique très important, qui améliore certainement l'estimation du taux de marge par rapport aux procédures qui n'en tiennent pas compte (Cette et Sylvain, 2001). D'autres éléments interviennent par ailleurs dans le mode de calcul du taux de marge (Sylvain, 2007), et ont un impact important sur son estimation.

(11) Prigent (1999) n'est en fait pas explicite sur cette question, et conclut seulement à l'existence d'une racine unitaire dans le processus engendrant la part des salaires, mais on comprend au vu de ses résultats que le processus est sans dérive. La part des salaires évolue sur la période étudiée selon une courbe en « $U$ » renversée, guère compatible avec l'existence d'un trend croissant ou décroissant.

(12) L'équation (2) est parfaitement compatible avec l'équation d'évaluation canonique de Harrison et Kreps (1979) et de Harrison et Pliska (1981), qui implique l'existence d'un facteur d'actualisation stochastique sous l'hypothèse d'absence d'opportunité d'arbitrage. Les taux d'actualisation qui apparaissent dans l'équation (2) sont liés aux facteurs d'actualisation stochastique, dont la définition précise dépend des hypothèses relatives aux préférences des agents. Ces taux ne sont pas égaux aux taux d'intérêt sans risque, car ils doivent comporter une prime de risque dont la spécification dépend des préférences que l'on prête aux agents.

(13) Le profit est à rapprocher de l'excédent brut d'exploitation (EBE) dans notre système de comptabilité.

(14) Ces paramètres sont positifs si la variable produit $\mathrm{q}_{\mathrm{t}} \mathrm{A}_{\mathrm{t}}$ est d'espérance positive et positivement autocorrélée. Si la variable produit est une différence de martingale, le terme $\alpha$ est nul.

(15) Pour avoir des séries aussi longues, nous avons effectué des collages de séries récentes avec des séries plus anciennes. Le prix des actions françaises est tiré, à partir de 1955, d'un indice large de l'OCDE (indice du mois de décembre de chaque année). Avant 1955, le prix des actions provient de la série « $\mathrm{Q} »$ du CEPII (présentée comme «un indice du cours des valeurs financières à revenu variable »). Le prix de la valeur ajoutée à partir de 1959 est constitué du déflateur du PIB français publié par l'OCDE. Avant 1959, nous avons utilisé la série "PPIBQ» du Cepii (prix de la PIB calculé par la production). Les séries (prix des actions et prix de la valeur ajoutée) sont en base 100 en l'an 2000. En conséquence, l'indice de prix réel du capital est de base unitaire en l'an 2000. Les sources utilisées sont le Cepii et l'OCDE : www.cepii.fr/francgraph/bdd/villa/mode.htm et webnet.oecd.org/wbos/

(16) Le test, avec un paramètre de troncature de valeur 4, a été appliqué sur la série avec terme constant (mais sans trend). Les statistiques $\hat{z}_{\alpha}$ et $\hat{z}_{t}$ apportent respectivement des corrections non paramétriques aux statistiques standard $T(\hat{\alpha}-1)$ et $(\hat{\alpha}-1) / \sqrt{V(\hat{\alpha})}$, où $\hat{\alpha}$ est le coefficient estimé de la variable retardée dans un test DF (Phillips et Perron, 1988).

(17) Le test de racine unitaire pose que l'hypothèse nulle est celle de non stationnarité. Il est conservateur envers cette hypothèse, alors que l'hypothèse préférée de notre point de vue est celle de stationnarité. Des probabilités critiques si peu élevées sont jugées en faveur de l'hypothèse préférée.

(18) Les paramètres sont estimés par la méthode du maximum de vraisemblance totale. L'estimateur de l'espérance par cette méthode diffère de la moyenne simple, car il tient compte de la dépendance des observations.

(19) Le début des années 40 a été marqué par une accélération très rapide de l'inflation, que les deux indices du prix des actions et du prix de la valeur ajoutée n'ont pas saisie avec la même vitesse, en raison d'une différence de synchronisme dans leur construction. L'inflation s'est ainsi retrouvée plus rapidement prise en compte dans l'indice financier, ce qui provoque le pic apparent de 1942. L'inflation est ensuite incorporée dans le déflateur du PIB, ce qui fait dégonfler cette fausse bulle.

(20) La méthode loess consiste à appliquer une régression polynomiale locale robuste sur le nuage de points. Nous avons utilisé un polynôme d'ordre deux, et un span (proportion de points définissant le degré de lissage) de 0,25 .

(21) La p-value de la pente du modèle (calculée sous l'hypothèse classique de sphéricité de la matrice de variance des erreurs) est de 4\%. Cela dit, il est évident que le modèle est mal spécifié (en raison d'une forte autocorrélation des erreurs). La correction des tests qui résulte de la prise en compte de l'autocorrélation des erreurs ne conduit cependant pas à changer les résultats. Le résultat de significativité de la pente est robuste.

(22) La bulle technologique de 2000 a ainsi été provoquée par l'attente d'une forte hausse de la productivité, dont notre modèle d'anticipation, basé sur l'hypothèse markovienne, ne peut rendre compte. L'équation (3) constitue une version opérationnelle trop simpliste de l'équation (2), et son estimation conduit certainement à sous-estimer la qualité du modèle.

(23) La p-value de la pente est négligeable (inférieure à 0,0001), attestant de la significativité du paramètre estimé. Cela dit, les mêmes remarques peuvent être faites que sur le modèle précédent : le modèle est mal spécifié (en raison notamment d'une forte autocorrélation des erreurs), mais les résultats des tests sont robustes. La variable explicative retardée n'est pas 
Aglietta M. (2008). Macroéconomie financière, Editions La Découverte.

Artus P. (2001). Politique monétaire, Economica.

Askenazy P. (2007). «Vers une théorie du partage de la valeur ajoutée », Actes $d u 11^{\text {ème }}$ colloque de l'Association de comptabilité nationale, Insee Méthodes, $\mathrm{n}^{\circ} 118$, pp. 161-170.

Beaubrun-Diant K.E. et Matheron J. (2008). «Rentabilités d'actifs et fluctuations économiques: une perspective d'équilibre général dynamique et stochastique ", Economie et Prévision, n 183-184, pp. 35-63.

Borio C. et Lowe P. (2002). « Asset Prices, Financial and Monetary Stability: Exploring the Nexus », document de travail, $\mathrm{n}^{\circ} 114$, BIS.

Cette G. et Sylvain A. (2001). «Partage primaire du revenu et rendement du capital: quelques repères empiriques pour plusieurs grands pays industrialisés $"$, Bulletin de la Banque de France, ${ }^{\circ}$ 93, septembre, pp. 73-89.

Cette G. et Szpiro D. (1988). « Durée de vie et âge moyen de l'outil de production », Economie et Statistique, $\mathrm{n}^{\circ} 208$, pp. 3-14.

Cleveland W.S. (1979). " Robust Locally Weighted Regression and Smoothing Scatterplots ", Journal of the American Statistical Association, $\mathrm{n}^{\circ} 368$, pp. 829-836.

Denison E.F. (1974). Accounting for United States Economic Growth, 1929-1969, The Brooking Institutions.

Dubois P. (1985). « Ruptures de croissance et progrès technique », Economie et statistique, $\mathrm{n}^{\circ} 181$, pp. 3-31.

Galbraith J.K. (1989). La crise économique de 1929 : anatomie d'une catastrophe financière, Payot.

Galbraith J.K. (1992). Brève histoire de l'euphorie financière, Editions du Seuil.

Harrison J.M. et Kreps D. (1979). « Martingales and Arbitrage in Multiperiod Securities Markets », Journal of Economic Theory, vol. 20, pp. 381-408.

Harrison M. et Pliska S. (1981). «Martingales and Stochastic Integrals in the Theory of Continuous Trading », Stochastic Processes and their Applications, vol. 11, pp. 215-260.

Jorgenson D.W., Gollop F.M. et Fraumeni B.M. (1987). Productivity and U.S. Economic Growth, Harvard University Press.

Kaldor N. (1963). «Capital accumulation and economic growth », in Lutz, F.A. et D.C. Hague (eds), Proceedings of a Conference Held by the International Economics Association, MacMillan.

Keynes J.M. (1936). Théorie générale de l'emploi de l'intéret et de la monnaie, trad. 1969, Editions Payot. 
Kindleberger C.P. (1978). Manias, Panics, and Crashes: History of Financial Crises, MacMillan.

Maddison A. (1995). L'économie mondiale 1820-1992, Analyse et statistiques, OCDE.

Phillips P.C.B. et Perron P. (1988). « Testing for a Unit Root in Time Series Regression », Biometrika, vol. 75, pp. 335-436.

Pollin J.-P. (2005). « Théorie de la politique monétaire : esquisses d'une refondation », Revue économique, vol. 56, pp. 507-539.

Prigent C. (1999). « La part des salaires dans la valeur ajoutée en France : une approche macroéconomique », Economie et statistique, ${ }^{\circ} 323$, pp. 73-94.

Sylvain A. (2001). "Rentabilité et profitabilité du capital : le cas de 6 pays industrialisés », Economie et Statistique, $\mathrm{n}^{\circ}$ 341-342, pp. 129-152.

Sylvain A. (2007). "Partage de la valeur ajoutée: éléments descriptifs et comparaisons internationales », Actes du $11^{\text {ème }}$ colloque de l'Association de comptabilité nationale, Insee Méthodes, $\mathrm{n}^{\circ} 118$, pp. 171-207.

Timbeau X. (2002). « Le partage de la valeur ajoutée en France », Revue de l'OFCE, n 80, pp. 63-85.

Williams J.B. (1938). The Theory of Investment Value, Harvard University Press. 